\title{
Interventricular Vessel of the Heart
}

\author{
Pekka J. Kuusela
}

\begin{abstract}
The communication from right to left through the interventricular septum of the heart became identified by the anatomical study for 4 years. One thousand nine hundred years ago, Galen stated that blood seeps through the perforations in the interventricular septum of the heart. However, William Harvey, working 400 years ago, failed to find any. The interventricular vessel is a slit between the fibers of the muscle feasible to be patent by relaxing and widening of the helical heart at the right atrial filling phase at the end of the diastole. The case exhibited the flow of venous blood passing from right to left through the interventricular vessel at the right atrial filling phase concordant to the fourth heart sound. The earliest left ventricular activation closed the interventricular sphincter surrounding the interventricular vessel in the middle of the left muscular part of the interventricular septum. The fourth heart sound is common at the atrial filling phase in hypertrophy of systemic hypertension and in ischemic heart disease. It is necessary to explore vigorously the unknown etiology of the fourth heart sound (S4).
\end{abstract}

Keywords: Interventricular vessel; Interventricular sinus; Interventricular sphincter; Fourth heart sound S4; Swiss cheese multiple muscular ventricular septal defect; Kuuselian vessel; Echocardiography

\section{Introduction}

The sinoatrial node initially activates the right atrium (RA), followed by activation of the left atrium (LA). Activation of the middle third of the left interventricular septum (IVS) occurs $5 \mathrm{~ms}$ before that of the other regions of the left ventricle (LV). The earliest epicardial breakthrough occurs at the anterior right epicardial surface near the apex, followed by anterior and posterior paraseptal areas of the LV [1, 2]. An abnormal fourth heart sound is common in hypertrophy of systemic hypertension and in ischemic heart disease, because the atrial "booster pump" is needed to assist the relatively stiff ischemic ventricle. The fourth heart sound disappears when coordinated atrial contraction ceases, as in atrial fibrillation

Manuscript submitted February 22, 2017, accepted February 14, 2018

Puijonkatu 27 A 22, 70100 Kuopio, Finland.

Email: PekkaJKuusela@luukku.com

doi: https://doi.org/10.14740/cr533w
$[3,4]$. The LV may develop negative pressure and sucking effect at the early diastole of the normal heart [5]. In the formation of the fetal muscular part of the IVS, the expanding ventricles grow and their medial walls approach and fuse, forming the septum. The inside corner between the septum and the right anterior ventricular wall exhibits the deep pits being called interventricular sinuses. The interventricular sinus passes through the right IVS formed from the medial wall of the expanding fetal right ventricle (RV). The opening of the interventricular vessel (kuuselian vessel) is located in the interventricular sinus between the medial walls of the expanding fetal RV and LV. The interventricular vessel is not a canal or channel or blood vessel, but a slit between the fibers of the muscle to the outer layer of the left central muscular part of the IVS and runs at an angle of about $90^{\circ}$ through the sphincter of the interventricular vessel and the left IVS into the LV. The interventricular vessel exhibits 2 - 3 oval 2 $\times 5 \mathrm{~mm}$ openings in the left central muscular part of the IVS surrounded by the interventricular sphincter. Agenesis of the interventricular sphincter in the middle of the left IVS may be the cause of the single muscular ventricular septal defect of the left central muscular part of the IVS, with numerous sieve like perforations through the right muscular part of the IVS [6]. The interventricular sphincter and the interventricular vessel are feasible to be patent by relaxing and widening of the helical heart at the right atrial filling phase at the end of the fetal diastole $[6,7]$. The left to right communication does not result as the earliest left ventricular activation closes the interventricular sphincter $[1,2]$.

\section{Case Report}

A retired male carpenter of 73 years age smoked between 25 and 55 years age. He had hypertension and the mild size nontoxic nodular struma for 10 years. He did not feel arythmia, dyspnea or breathing difficulties with frost. He felt sweating when sleeping and reading on his back for 10 years. He felt also upper abdominal swelling and dull pain.

Walking and working by his hands relieved the symptom. He was not a diabetic or obese. The blood pressure elevated and became more labile. He used medicines carvedilol $12.5 \mathrm{mg} \times$ 2, valsartan $160 \mathrm{mg} \times 1$, and acetylsalicylic acid $100 \mathrm{mg} \times 1$.

Blood pressure was 190 - 160/120 - $100 \mathrm{~mm} \mathrm{Hg}$. He exhibited blue lips, mild systolic bruit over the ascending aorta, no bruit on the neck, abdomen and thighs, cool feet, palpable arteries on the both feet, no swelling, and no palpable lymph nodes.

Hemoglobin was $180 \mathrm{~g} / \mathrm{L}(134$ - 167), hematocrit was 0.53 


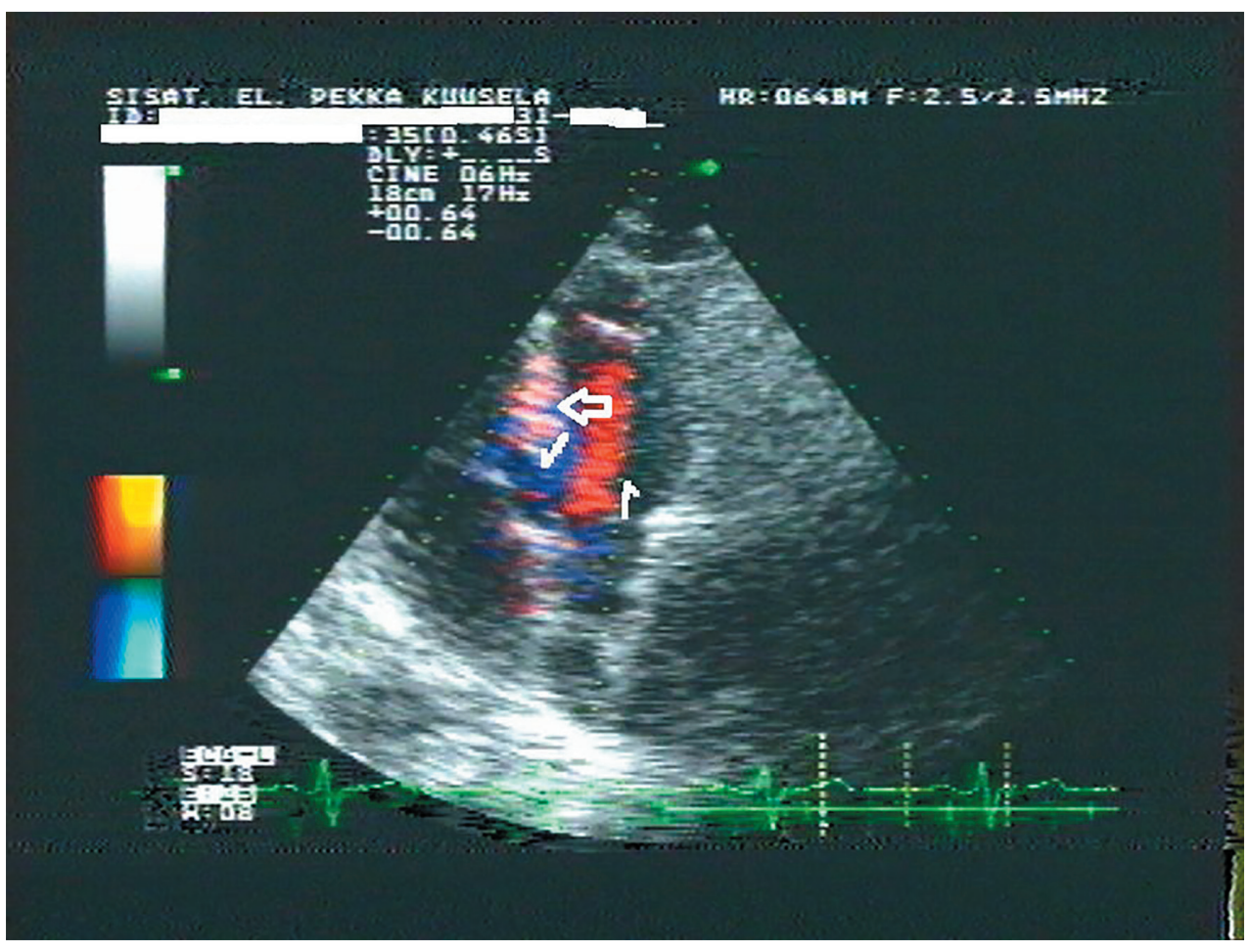

Figure 1. The right atrial filling phase was toward the apex of the RV on the right side of the septal cusp of the tricuspid valve (TV) (right arrow). The flow was again between the septum and the septal cusp of the TV toward the interventricular sinus (left arrow). The flow was from right to left through the interventricular sphincter into the LV (high arrow).

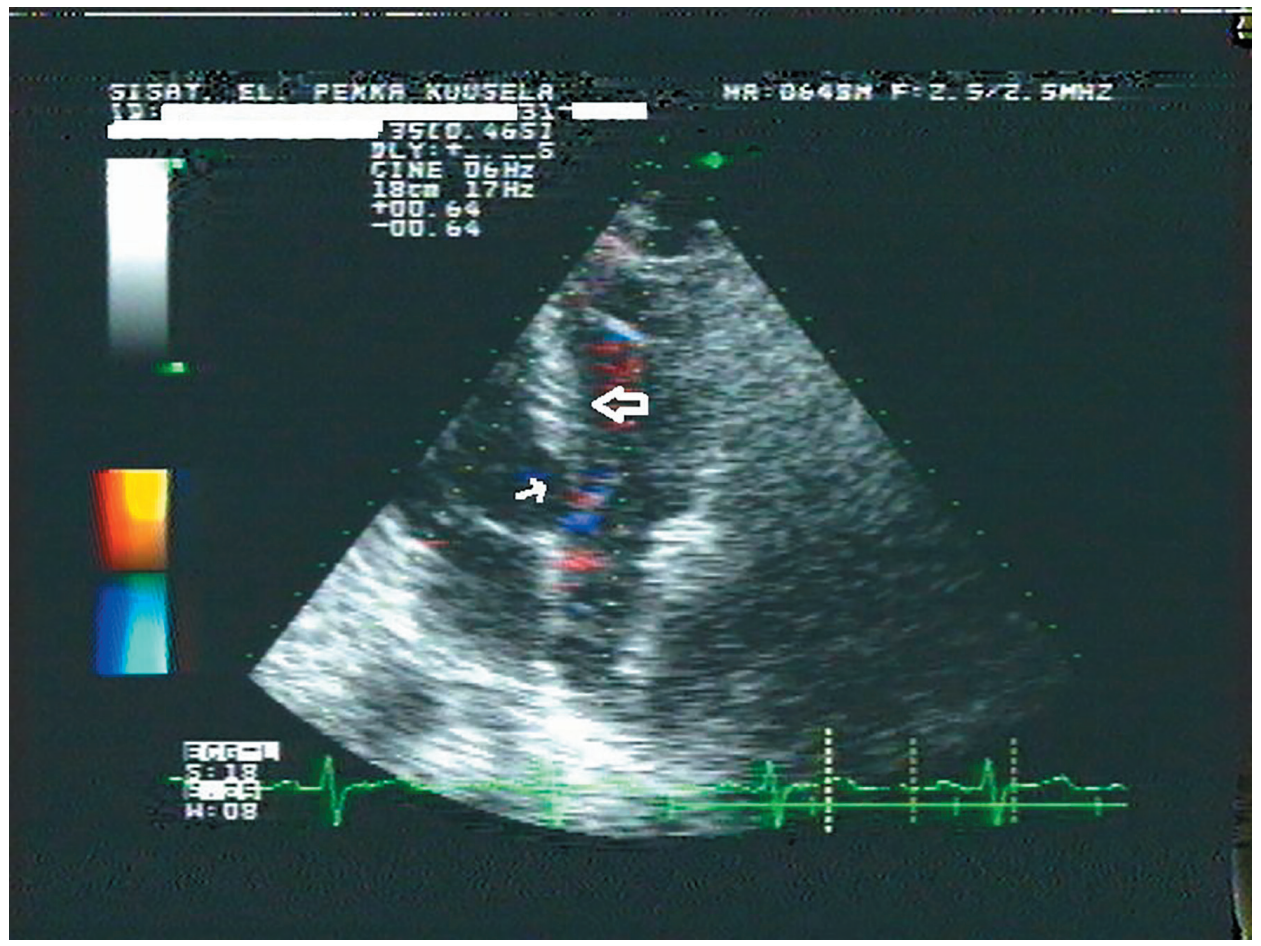

Figure 2. The end of the diastole $0.12 \mathrm{~s}$ later than Figure 1. The limit was visible between the right and the left muscular part of the IVS formed of the opposing fetal medial walls of the RV and the LV (low arrow). The openings of the interventricular vessel (kuuselian vessel) into the LV were visible between the interventricular sphincter in the left central muscular part of the IVS (high arrow). 


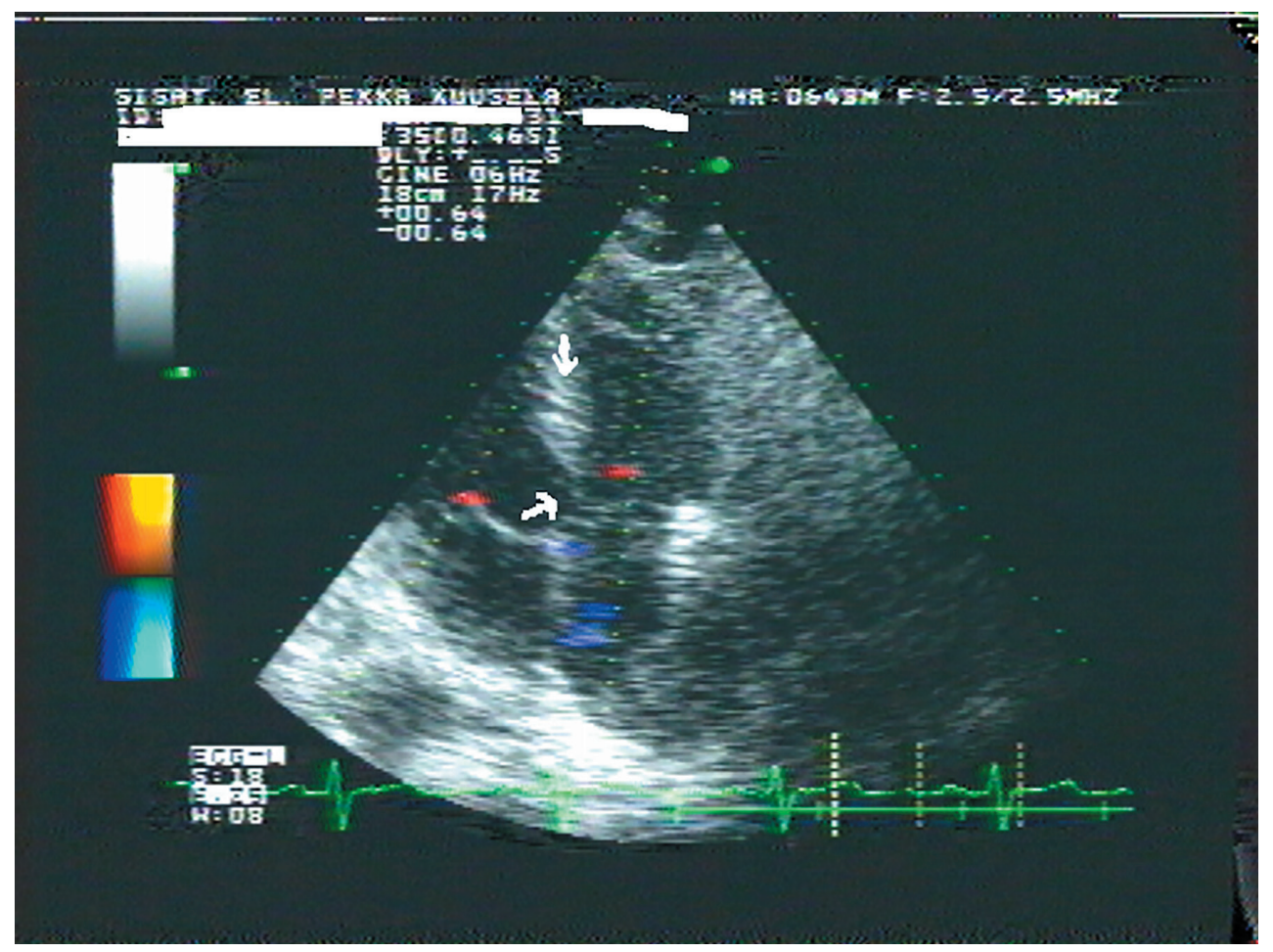

Figure 3. The limit between the right and the left muscular part of the IVS disappeared and the interventricular vessel right to the interventricular sphincter closed concordant to activation of the RV (low arrow) [1]. The interventricular sphincter exhibited closing movement concordant to activation of the middle third of the left IVS (high arrow) [1, 2].

(0.39 - 0.50), MCV was 85 (82 - 98), MCH was 29 (27 - 33), MCHC was 344 (315 - 360), and THROMB was 290 (150 $360)$. TSH was $0.10 \mathrm{mU} / \mathrm{L}(0.40-5.00)$, T4V was $4.0 \mathrm{pmol} / \mathrm{L}$ (8.0 - 20.00), T3-V was 6.6 pmol/L (4.4 - 7.7), S-ALT was 33 $\mathrm{U} / \mathrm{L}(0$ - 50), S-creatinine was $78 \mu \mathrm{mol} / \mathrm{L}(75$ - 105), S-Na was $141 \mathrm{mmol} / \mathrm{L}$ (134 - 144), S-K was $4.2 \mathrm{mmol} / \mathrm{L}(3.4-4.7)$, and S-cholesterol was $5.1 \mathrm{mmol} / \mathrm{L}$ (3.6 - 5.0). HbA1c 5.8 was normal. He exhibited secondary polycytemia and low TSH with euthyroidism. ECG: sinus rhythm, P-R interval was $184 \mathrm{~ms}$, ventricular extrasystoles, LAHB. V1 PTF $-1.0 \mathrm{~mm} \times 0.07 \mathrm{~s}$ $=-0.07 \mathrm{mms}$. P-duration was more than $100 \mathrm{~ms}$. He exhibited the left atrial load. Thorax X-ray showed the heart and lungs were normal. The upper abdomen ultrasound by Aloka SSD-650 with $3.5 \mathrm{MHz}$ convex sector transducer showed normal size kidneys $11.3 / 10.6 \mathrm{~cm}$. Cortexes was 19/17 mm. Liver, gallbladder, choledochus and pancreas were normal. Echocardiography by Aloka SSD-870 with $2.5 \mathrm{MHz}$ and 2.5/2.0 MHz sector transducers and U-matic videorecorder showed LA 37, IVSh 7, LVd 60 (56), PWh 7mm, EF 56\%, mitral E/A 29/63 reduced, aortic valve regurgitant area $0.3 \mathrm{~cm}^{2}$, RVd 28 , normal right ventricular eccentricity index 1.0, RVSP $29.2 \mathrm{~mm}$ $\mathrm{Hg}$ (20), and PA $24 \mathrm{~mm}$. The patient exhibited the stiff and enlarged LV with the mild aortic valve regurgitation and elevated right ventricular systolic pressure. The heart exhibited at the right atrial filling phase the flow of venous blood passing from right to left through the interventricular vessel into the LV. The figures and the video demonstrated the pathophysiological function of the interventricular vessel (Figs. 1-4) (Supplementary video 1 , see in www.cardiologyres.org. The venous flow from right to left through the interventricular vessel of the heart appeared at the right atrial systole. The venous flow was moving towards the left outflow tract in the LV. The limit between the right and the left muscular part of the IVS right to the interventricular sphincter closed previous to the interventricular sphincter. The heart was moving and the limit was visible in a small volume of the interventricular septum. The limit was interventricular vessel of the heart between the right and the left muscular part of the IVS). The figures will be more informative together with the Reference [6]. That is an open anatomical study.

\section{Discussion}

The flow from right to left through the muscular part of the IVS at the right atrial filling phase suggested low left ventricular diastolic pressure and was concordant to the interventricular vessel (kuuselian vessel) and to widening of the helical heart at the end of the diastole by ventricular band relaxation [5-7]. The interventricular vessel right to the interventricular sphincter closed concordant to activation of the RV. The interventricular sphincter began closing at the early systole concordant to activation of the left middle third of the IVS $[1,2]$. The flow from right to left through the interventricular vessel into the LV at the right atrial filling phase was concordant to the abnormal fourth heart sound $[3,4]$. Hypoxia may be the physiological factor to recruit the interventricular vessel of the fetal heart 


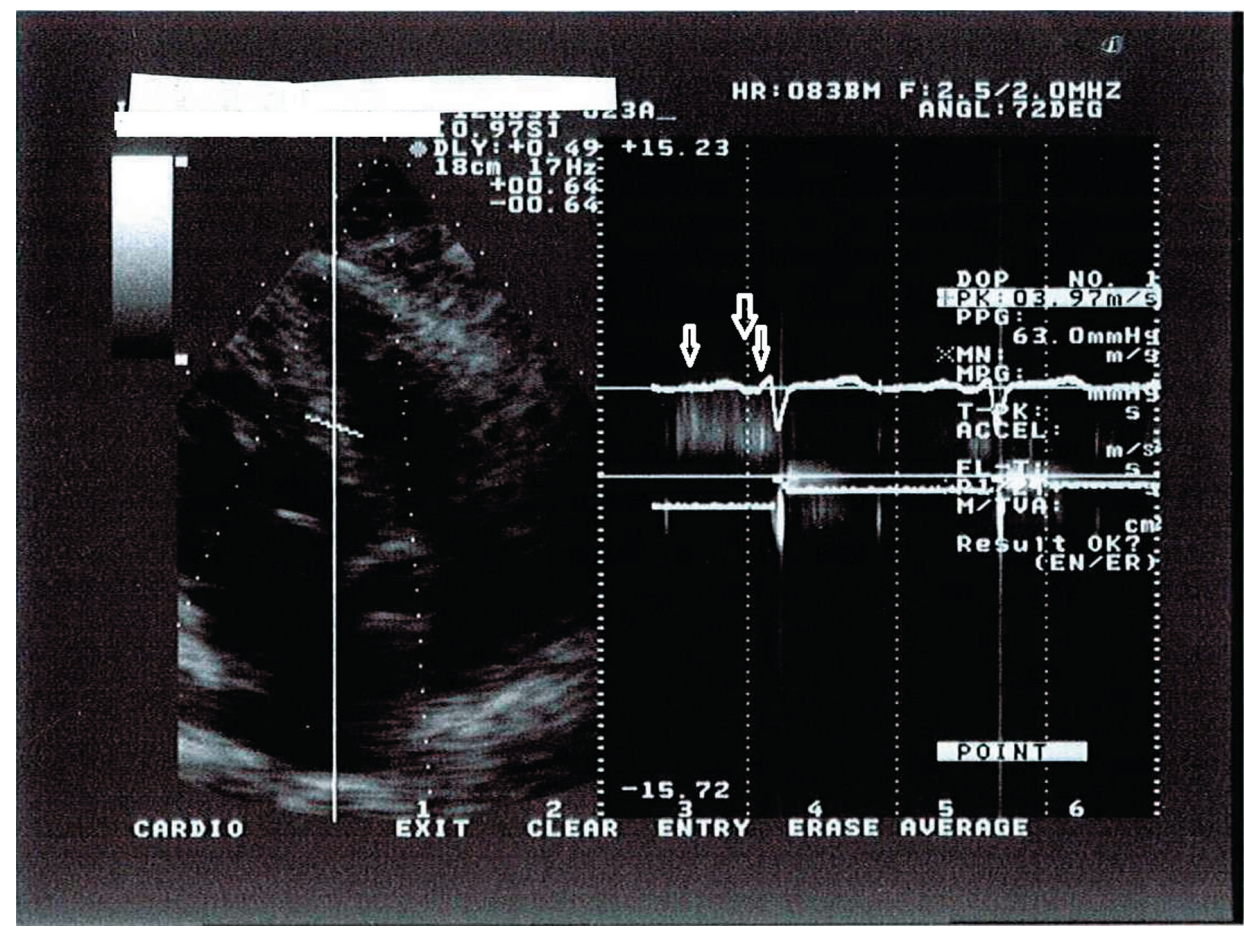

Figure 4. The CW Doppler recorded noise caused by two to three streams of blood close to each other through the openings of the interventricular vessel into the LV to demonstrate the flow at the diastole but not to measure the gradient. The gradient was not $63 \mathrm{~mm} \mathrm{Hg}$ but a flow artifact. The flow through the interventricular vessel into the LV at the middle diastole suggested low left ventricular diastolic pressure (left arrow) [5]. The flow into the LV at the right atrial filling phase was concordant to the fourth heart sound (S4) (high arrow) [3, 4]. The interventricular vessel closed with a dense signal of flow as the blood ran off the interventricular vessel into the LV at the very early systole. The velocity was about $2.3 \mathrm{~m} / \mathrm{s}$ and the gradient was less than $23 \mathrm{~mm}$ $\mathrm{Hg}$ (right arrow) [1, 2, 6].

and augment the flow of the oxygenated blood from right to left. The patient exhibited secondary polycythemia very likely recruited by hypoxia. The fourth heart sound is common in hypertrophy of systemic hypertension and in ischemic heart disease. It is necessary to explore vigorously the unknown etiology of the fourth heart sound (S4). The embryological and anatomical basis of the single muscular ventricular septal defect of the left central muscular part of the IVS, with numerous sieve like perforations through the right muscular part of the IVS formed from the medial wall of the fetal RV should be considered. Aloka 870 was the modern system for 27 years. Today the modern ultrasound imaging systems have clearer and sharp imaging. The cardiologist will have better imaging quality than the author not only by the modern imaging system but also by keeping one's mind on imaging and studying anatomy of the heart.

\section{Source of Funding}

None.

\section{Disclosures}

None.

\section{References}

1. Durrer D, van Dam RT, Freud GE, Janse MJ, Meijler FL, Arzbaecher RC. Total excitation of the isolated human heart. Circulation. 1970;41(6):899-912.

2. Riera AR, Uchida AH, Schapachnik E, Dubner S, Zhang L, Filho CF, Ferreira C, et al. The history of left septal fascicular block: chronological considerations of a reality yet to be universally accepted. Indian Pacing Electrophysiol J. 2008;8(2):114-128.

3. Aronow WS, Papageorge's NP, Uyeyama RR, Cassidy J. Maximal treadmill stress test correlated with postexercise phonocardiogram in normal subjects. Circulation. 1971;43(6):884-888.

4. Perloff JK, Braunwald E. Physical examination of the heart and circulation. In Braunwald E (ed), Heart Disease, fifth ed. WB Saunders, Philadelphia, 1997. p. 3435 .

5. Udelson JE, Bacharach SL, Cannon RO, Bonow RO. Minimum left ventricular pressure during beta-adrenergic stimulation in human subjects. Evidence for elastic recoil and diastolic "suction" in the normal heart. Circulation. 1990;82(4):1174-1182.

6. Kuusela PJ. The heart exhibits right to left communication between the fibres of the muscular part of the interventricular septum. Folia Morphol (Warsz). 2014;73(1):42- 
50.

7. Torrent-Guasp F, Buckberg GD, Clemente C, Cox JL, Coghlan HC, Gharib M. The structure and function of the helical heart and its buttress wrapping. I. The normal macroscopic structure of the heart. Semin Thorac Cardiovasc Surg. 2001;13(4):301-319. 